\title{
Transient Cerebral Ischemic Attack and Delirium Heralding the Vasculitic Phase of Eosinophilic Granulomatosis With Polyangiitis (Churg-Strauss Syndrome)
}

\author{
Paolo Falsetti ${ }^{\mathrm{a}, \mathrm{c}}$, Caterina Acciai ${ }^{\mathrm{a}}$, Rosanna Palilla ${ }^{\mathrm{a}}$, Giovanni Linoli ${ }^{\mathrm{b}}$, Lucia Lenzi ${ }^{\mathrm{a}}$
}

\begin{abstract}
Eosinophilic granulomatosis with polyangiitis (EGPA), formerly known as Churg-Strauss syndrome, is a rare systemic vasculitis characterized by a prodromic history of allergic asthma and sinusitis for many years, followed by an eosinophilic phase and a vasculitic phase. Central nervous system (CNS) involvement associated with EGPA is very rare, in particular as first sign of the vasculitic phase. We describe a patient with transient cerebral ischemic attack and delirium heralding the vasculitic phase of EGPA. We report a case of EGPA (Churg-Strauss syndrome) in a 76-year-old Italian woman with acute neurologic and neuropsychiatric symptoms and history of asthma and sinusitis. She was admitted in stroke unit with acutely developing aphasia, ataxia and delirium without acute lesions on neuroimaging. When she was transferred in neurorehabilitation unit, an antineutrophil cytoplasmic antibodies (ANCA)-associated vasculitis was suspected on the basis of anamnesis, facies (saddle nose) and presence of systemic inflammation. She was finally diagnosed with EGPA because of the presence of subclinical demyelinating polyneuropathy, interstitial pulmonary disease and increased myeloperoxidase-specific ANCA (MPO-ANCA). The patient's neurologic symptoms were, therefore, attributed to a vasculitic process, and they improved with steroid treatment. CNS involvement associated with EGPA is very rare and, in this case it represented the first sign of systemic vasculitis after many years of prodromic phase, and it should be considered in patients with acute cerebrovascular disease associated with systemic inflammation or eosinophilia and anamnestic asthma or sinusitis.
\end{abstract}

Keywords: ANCA; Cerebrovascular disease; Churg-Strauss syndrome; EGPA; Vasculitis

\section{Introduction}

Eosinophilic granulomatosis with polyangiitis (EGPA), for-

Manuscript accepted for publication September 17, 2015

${ }^{a}$ Neurorehabilitation Unit, S. Donato Hospital, Arezzo, Italy

${ }^{\mathrm{b} S}$ Stroke Unit, S. Donato Hospital, Arezzo, Italy

${ }^{\mathrm{c} C}$ Corresponding Author: Paolo Falsetti, Neurorehabilitation Unit, San Donato

Hospital, via Nenni 52100, Arezzo, Italy. Email: paolo.falsetti@virgilio.it

doi: http://dx.doi.org/10.14740/jmc2300w merly known as Churg-Strauss syndrome, is a rare systemic vasculitis included in the spectrum of anti-neutrophil cytoplasmic antibodies (ANCA)-associated vasculitis (AAV). EGPA is typically characterized by a prodromic history of allergic asthma, sinusitis and nasal polyposis for many years, followed by an eosinophilic phase hallmarked by peripheral eosinophilia and organ involvement, and a vasculitic phase with clinical manifestations due to small-vessel vasculitis [1-4].

Central nervous system (CNS) involvement in EGPA is much less frequently reported than peripheral neuropathy in the literature, but it is one of the major causes of morbidity and mortality [1-6]. Therefore, prompt diagnosis and treatment are mandatory when EGPA is associated with cerebral involvement.

We describe a patient with transient cerebral ischemic attack and delirium heralding the vasculitic phase of EGPA.

\section{Case Report}

A 76-year-old Italian woman with a history of asthma, sinusitis, mild renal insufficiency and arthralgias was admitted in Stroke Unit of San Donato Hospital in Arezzo (Tuscany, Italy) with acutely developing aphasia, ataxia and delirium. National Institutes of Health Stroke Scale score was 14. She was outside recombinant tissue plasminogen activator treatment window. The patient was afebrile.

Laboratory data at admission revealed C-reactive protein (CRP) $1.7 \mathrm{mg} / \mathrm{dL}$ (normal value $(\mathrm{NV})<0.5$ ), erythrocyte sedimentation rate (ESR) $51 \mathrm{~mm} / \mathrm{h}(\mathrm{NV} 2$ - 30), fibrinogen 461 $\mathrm{mg} / \mathrm{dL}$ (NV 150 - 450), creatinine $1.35 \mathrm{mg} / \mathrm{dL}$ (NV 0.50 - 1.20) with a glomerular filtration calculated by CKD-EPI was 38 $\mathrm{mL} / \mathrm{min}$, and mild proteinuria was $30 \mathrm{mg} / \mathrm{dL}(\mathrm{NV}<15)$ in urinalysis. Complete blood count showed a significant increase of eosinophils (11\%), with abnormal blood cell count $(12,000)$, hemoglobin $10.6 \mathrm{~g} / \mathrm{dL}$ (NV 11.7 - 16), with normal TSH (1.55 $\mathrm{mUI} / \mathrm{L}, \mathrm{NV} 0.20$ - 4.20) and complement.

She had some risk factors for stroke: cholesterol $299 \mathrm{mg} /$ dL $(\mathrm{NV}<200)$, with non-HDL $221 \mathrm{mg} / \mathrm{dL}(\mathrm{NV}<130)$ and homocysteine $34.2 \mu \mathrm{mol} / \mathrm{L}$ (NV 0 - 15). Moreover, she was treated for hypertension.

In anamnesis 6 months before she underwent rheumatological visit for her symmetrical arthralgias associated with recurrent fever. She was treated with a low corticosteroid dose 

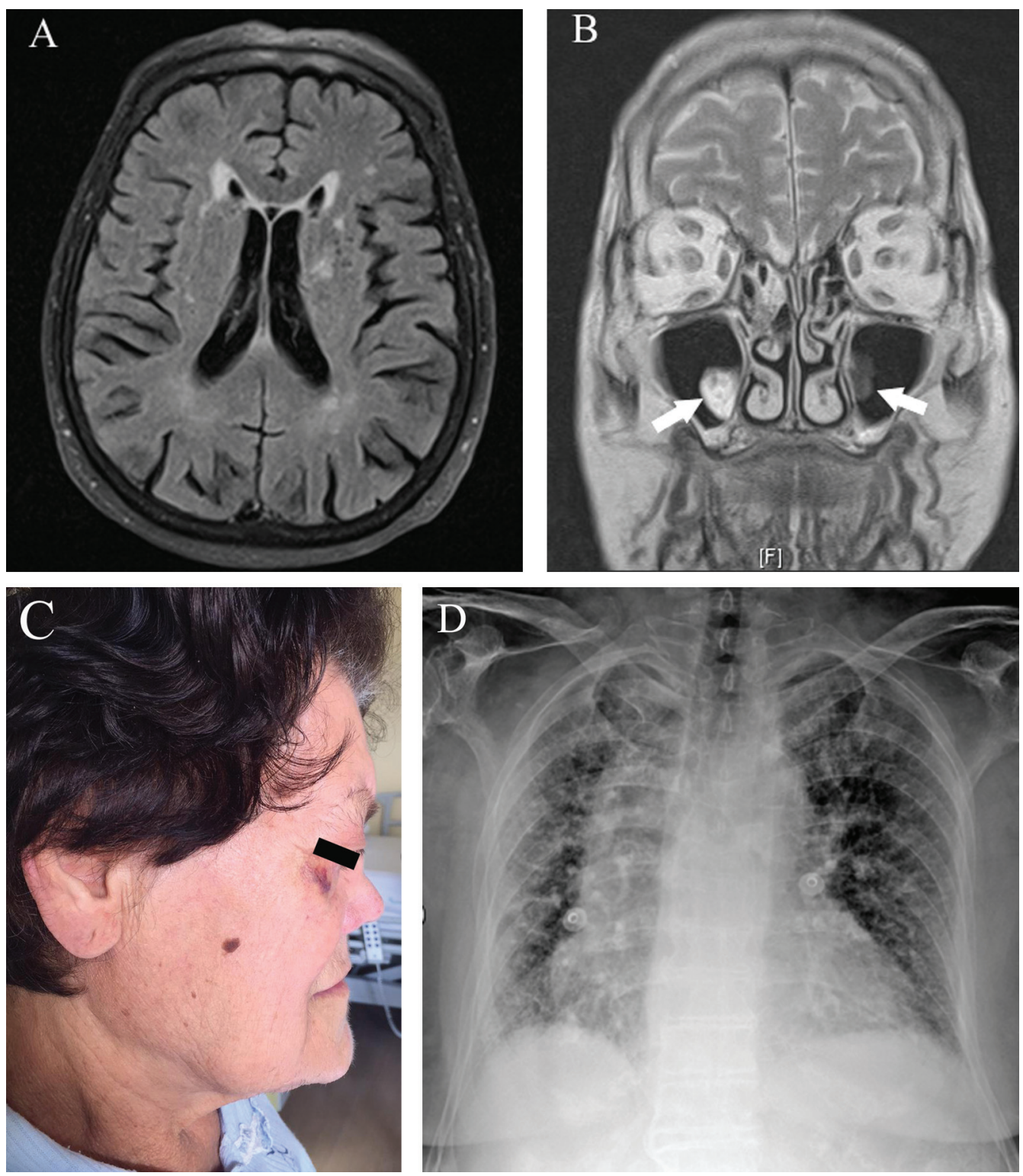

Figure 1. (A) Brain MRI showed multiple hyperintense lesions in both corona radiata and the bilateral deep periventricular white matter on the T2 FLAIR. The lesions showed hypointensity on DWI (not shown). (B) Head MRI showed bilateral maxillary sinuses non-erosive polyposis (white arrows) on T2 coronal scan. (C) The peculiar facies of the patient with a saddle nose acquired deformity and mild exophthalmos. (D) The chest radiograph shows peripheral and symmetrical patchy consolidation in the lungs.

(8 $\mathrm{mg}$ of methylprednisolone) with suspicion of polymyalgia rheumatica. The corticosteroid therapy was effective on arthralgias and fever, and the follow-up was recently interrupted.

Brain computed tomography (CT) at admission showed diffuse cerebrovascular disease and excluded cerebral hemorrhage. Magnetic resonance imaging (MRI) showed signs of diffuse small-vessel cerebrovascular disease in basal nuclei and periventricular white matter with no acute focal lesion on diffusion-weighted imaging (DWI) and paranasal maxillary sinuses non-erosive polyposis (Fig. 1A, B).

CT-angiography showed bilateral but not critical internal carotid arteries stenosis. The diagnostic approach for ischemic stroke failed to reveal a definite etiology, and she was treated with clopidogrel $75 \mathrm{mg}$ for secondary stroke prevention.

The patients was, therefore, admitted in neurorehabilitation unit, a post-intensive unit of the same hospital, with dysarthria, with moderate cognitive impairment (memory loss, disorientation), and ill-defined but symmetrical pain and weakness in her legs and ataxia, unable to walk. The rheumatologist noted the peculiar facies of the patient that showed a saddle nose acquired deformity, and mild exophthalmos (Fig. 1C). The patient and her parents confirmed that such deformities were observed progressively after repeated sinusitis, about 10 years before. Moreover, she told that in the past she was also admitted in a pneumologic clinic to treat severe asthma and sinusitis (clinic documentation was unavailable). 
The rheumatologist re-evaluated the complete anamnesis of the patient and the diagnosis of ANCA-associated vasculitis, or relapsing polychondritis was then suspected.

Indirect immuno-fluorescence showed a p-ANCA pattern. Enzyme-linked immunosorbent assay (ELISA) confirmed the positivity of myeloperoxidase ANCA (MPO-ANCA) at 17.87 $\mathrm{UI} / \mathrm{mL}(\mathrm{NV}<5)$. Anti-nuclear antibodies (ANA) and anti-extractable nuclear antigens (ENA) and rheumatoid factor (RF) resulted negative. Abnormal level of total $\operatorname{IgE}$ was also noted $1,355 \mathrm{UI} / \mathrm{mL}$ (NV 0 - 100).

The patient underwent laryngoscopy that excluded subglottic narrowing. Echocardiography excluded cardiomyopathy, endomyocarditis, valvular heart disease and pericarditis.

Chest X-ray revealed diffuse and symmetrical interstitial pulmonary disease with a restrictive pattern of functional tests (Fig. 1D).

An EMG confirmed a mixed motor and sensory axonal and demyelinating polyneuropathy.

A comprehensive anamnestic and clinical examination permitted to diagnose EGPA on the basis of American College of Rheumatology (1990) criteria [7]. The diagnosis was probable, as the patient fulfilled five of the six criteria at the time of present admission or anamnestic (asthma, eosinophilia $>10 \%$, polyneuropathy, pulmonary infiltrates, paranasal sinus abnormality). Furthermore, based on the elevated serum titer of $p$ ANCA and total IgE and nose chondritis, the patient was diagnosed as having EGPA, although biopsy was not performed.

On the suspicion that the patient had a vasculitic CNS involvement, the patient was given $32 \mathrm{mg}$ of methylprednisolone orally every day, and with gradual tapering. Weakness and pain in both lower extremities improved slowly but continuously, and she became much less confused with 2 weeks of treatment. The patient was discharged after about 1 month on $8 \mathrm{mg}$ methylprednisolone daily. Inflammatory reactant was negative. She was able to walk with mild assistance, without relevant speech or cognitive impairment.

\section{Discussion}

EGPA is a systemic, small-vessel necrotizing vasculitis that occurs in several organ systems, but it rarely affects the CNS $[2,3,5,6]$.

Involvement of small intracranial vessels is more likely to result in transient cerebral ischemic attack, cerebral infarction or hemorrhage, and CNS involvement of EGPA may cause significant morbidity and mortality $[1,5]$. Because of the rarity of CNS involvement, and in particular as first presentation of the vasculitic phase, the suspicion of such rare vasculitis in patients with acute cerebrovascular disease can be challenging $[2,3,8]$.

An Italian long-term follow-up study of 38 patients affected with EGPA did not report case of CNS involvement, and the recent study of the French Vasculitis Study Group cohort reported a 5.2\% $[2,3]$. CNS involvement in EGPA seems to be more frequent in Asian patients than in Caucasian [6].

However, the presence of high titer of ANCA hallmarks the vasculitic disease complications, as some large studies demon- strated that ANCA were positive in among 30-40\% of EGPA patients and that ANCA-positive patients more frequently had peripheral neuropathy, glomerulonephritis, purpura and CNS involvement (which are due to small-vessel vasculitis) [1, 9, 10]. Thus ANCA should be tested in patients with cerebrovascular disease associated with systemic inflammation or eosinophilia and anamnestic asthma.

The present case illustrates that EGPA is a risk factor for multiple cerebral infarctions and should be considered in differential diagnosis of patients with cerebrovascular disease symptoms, systemic inflammation and asthma with hypereosinophilia.

Early detection of this condition and treatment with immunosuppressive therapy can lead to improved systemic and neurologic function and prevent long-term disability.

In conclusion, CNS involvement of EGPA is very rare, but it may cause significant morbidity and mortality. EGPA should be suspected, and ANCA should be tested in patients with acute cerebrovascular disease associated with systemic inflammation or eosinophilia and anamnestic asthma or sinusitis.

\section{Conflict of Interest}

Authors declare no financial or personal relationships that may pose conflict of interest.

\section{References}

1. Vaglio A, Buzio C, Zwerina J. Eosinophilic granulomatosis with polyangiitis (Churg-Strauss): state of the art. Allergy. 2013;68(3):261-273.

2. Baldini C, Della Rossa A, Grossi S, Catarsi E, Talarico R, d'Ascanio A, Mosca M, et al. [Churg-Strauss syndrome: outcome and long-term follow-up of 38 patients from a single Italian centre]. Reumatismo. 2009;61(2):118-124.

3. Comarmond C, Pagnoux C, Khellaf M, Cordier JF, Hamidou M, Viallard JF, Maurier F, et al. Eosinophilic granulomatosis with polyangiitis (Churg-Strauss): clinical characteristics and long-term followup of the 383 patients enrolled in the French Vasculitis Study Group cohort. Arthritis Rheum. 2013;65(1):270-281.

4. Mouthon L, Dunogue B, Guillevin L. Diagnosis and classification of eosinophilic granulomatosis with polyangiitis (formerly named Churg-Strauss syndrome). J Autoimmun. 2014;48-49:99-103.

5. Sehgal M, Swanson JW, DeRemee RA, Colby TV. Neurologic manifestations of Churg-Strauss syndrome. Mayo Clin Proc. 1995;70(4):337-341.

6. Sada KE, Amano K, Uehara R, Yamamura M, Arimura Y, Nakamura Y, Makino H. A nationwide survey on the epidemiology and clinical features of eosinophilic granulomatosis with polyangiitis (Churg-Strauss) in Japan. Mod Rheumatol. 2014;24(4):640-644.

7. Masi AT, Hunder GG, Lie JT, Michel BA, Bloch DA, Arend WP, Calabrese LH, et al. The American College of Rheumatology 1990 criteria for the classification of Churg-Strauss syndrome (allergic granulomatosis and an- 
giitis). Arthritis Rheum. 1990;33(8):1094-1100.

8. Tanaka K, Koga M, Ishibashi-Ueda H, Matsumoto C, Toyoda K. Churg-Strauss syndrome with concomitant occurrence of ischemic stroke and relapsing purpura. J Stroke Cerebrovasc Dis. 2012;21(8):911 e919-910.

9. Oka N, Kawasaki T, Matsui M, Shigematsu K, Unuma T, Sugiyama H. Two subtypes of Churg-Strauss syndrome with neuropathy: the roles of eosinophils and ANCA. Mod Rheumatol. 2011;21(3):290-295.

10. Sinico RA, Di Toma L, Maggiore U, Bottero P, Radice A, Tosoni C, Grasselli C, et al. Prevalence and clinical significance of antineutrophil cytoplasmic antibodies in ChurgStrauss syndrome. Arthritis Rheum. 2005;52(9):29262935. 